\title{
The bivalency effect: adjustment of cognitive control without response set priming
}

\author{
Alodie Rey-Mermet • Beat Meier
}

Received: 25 October 2010/Accepted: 14 February 2011/Published online: 24 February 2011

(C) Springer-Verlag 2011

\begin{abstract}
The occasional occurrence of bivalent stimuli, that is, stimuli with features relevant to two tasks, slows performance on subsequent tasks with univalent stimuli, including those which have no common features with bivalent stimuli (i.e., the "bivalency effect"). We have suggested that the bivalency effect might stem from an episodic context binding arising from the occasional occurrence of bivalent stimuli. However, as the same response set is used usually for univalent and bivalent stimuli, bivalent stimulus features may be negatively primed via response features. We investigated this possibility in two experiments, in which one group of participants used the same response keys for all tasks and another group used separate response keys. The results showed a comparable bivalency effect in both groups. Thus, it rather results from episodic context binding than from response set priming.
\end{abstract}

\section{Introduction}

Performance in response to bivalent stimuli, i.e., stimuli with features relevant to two different tasks, is slowed compared to univalent stimuli (e.g., Jersild, 1927; Meiran, 2008; Steinhauser \& Hübner, 2009). Even when only a few bivalent stimuli are presented among tasks with univalent stimuli, performance is also slowed on subsequent univalent trials. Moreover, this slowing, coined the bivalency effect, occurs for tasks with univalent stimuli that share relevant stimulus features with bivalent stimuli as well as

\footnotetext{
A. Rey-Mermet · B. Meier $(\square)$

Department of Psychology, University of Bern,

3000 Bern 9, Switzerland

e-mail: beat.meier@psy.unibe.ch
}

for tasks with univalent stimuli that share no relevant stimulus features with bivalent stimuli (Meier, Woodward, Rey-Mermet, \& Graf, 2009; Rogers \& Monsell, 1995; Woodward, Meier, Tipper, \& Graf, 2003; Woodward, Metzak, Meier, \& Holroyd, 2008; Wylie \& Allport, 2000). We have suggested that the bivalency effect might stem from an episodic context binding arising from the occasional occurrence of bivalent stimuli (Meier et al., 2009). However, as we have investigated the bivalency effect only with the same response features for all univalent and bivalent stimuli, bivalent stimulus features might still interfere with the processing of univalent trials that share relevant response features with the bivalent stimuli (Hommel, 2004; Metzker \& Dreisbach, 2009). Thus, bivalent stimulus features would be negatively primed over these trials. This would slow performance and result in the bivalency effect. The purpose of the present study was to test whether the bivalency effect might result from this kind of negative response set priming.

In the previous studies investigating the bivalency effect (Meier et al., 2009; Woodward et al., 2003; 2008), participants were asked to perform three binary tasks in a given order, such as a parity decision (odd vs. even numerals), a colour decision (red vs. blue shapes), and a case decision (uppercase vs. lowercase letters). Most stimuli were univalent (i.e., black numerals for the parity decision, coloured shapes for the colour decision, and black letters for the case decision). However, on a few trials of one task, the stimuli were made bivalent (e.g., by presenting the letters for the case decisions in either blue or red print colour). The results consistently showed a performance slowing for all tasks following bivalent stimuli, including those with stimuli that shared no relevant stimulus features with the bivalent stimuli (i.e., the parity decisions). This finding - the bivalency effect-is robust, 
occurring with different types of tasks (parity, colour, case, size, vowel/consonant), with different types of bivalent stimuli (coloured or large/small letters) and with different modalities (visual, auditory), and it is enduring (lasting more than $20 \mathrm{~s}$ after a bivalent stimulus; Meier et al. (2009)). Furthermore, in a study using functional magnetic resonance (Woodward et al., 2008), the bivalency effect was associated with activation in the dorsal anterior cingulate cortex (dACC), a brain area recruited for the adjustment of cognitive control (Botvinick, Braver, Barch, Carter, \& Cohen, 2001).

Together, these studies suggest that the bivalency effect reflects a robust adjustment of cognitive control, which is recruited to fine-tune performance according to the occasional occurrence of bivalent stimuli. This cognitive control effect challenges current task-switching theories as well as theories of cognitive control that focus primarily on processes operating across stimulus and task representations. For instance, Allport et al. (Allport, Styles, \& Hsieh, 1994; Allport \& Wylie, 1999; 2000; Wylie \& Allport, 2000) proposed a negative priming account in which bivalent stimulus features led to the persisting suppression or inhibition of the previously active task-set. In the bivalency effect paradigm outlined above, it would mean that bivalent stimulus features interfere with the processing of the univalent trials that share relevant stimulus features with the bivalent stimuli. Thus, bivalent stimulus features would be inhibited and negatively primed. For example, when coloured letters-the bivalent stimuli-are encountered in case decisions, the particular stimulus features (i.e., case and colour) would be bound together. When the colour decision is encountered later, the colour feature would automatically activate the case feature. The irrelevant activation of these stimulus features interferes with trial processing and slows down performance on the colour decision. Similarly, when the case decision is encountered, the colour feature is also activated. Accordingly, a negative priming account can explain the slowing that was found on tasks with univalent stimuli sharing relevant stimulus features with the bivalent stimuli (i.e., the colour and case decisions). However, it cannot explain slowing that was found on tasks with univalent stimuli sharing no relevant stimulus features with the bivalent stimuli (i.e., the parity decision).

Similarly, according to a task-decision process account, those univalent stimuli which share stimulus features with the bivalent stimuli, might require a task-decision process (Braverman \& Meiran, 2010; see also Fagot, 1994; Meiran, Kessler, \& Adi-Japha, 2008; Monsell, Yeung, \& Azuma, 2000; Rogers \& Monsell, 1995; Rubinstein, Meyer, \& Evans, 2001; Sohn \& Anderson, 2001). More precisely, they might cue the irrelevant task and thus would slow down the task-decision process. For example, when bivalent stimuli are coloured letters, the stimuli for the colour decision would also cue the case decision. Accordingly, an additional process would be required to resolve this ambiguity and to select the colour decision as relevant. Similarly, the stimuli for the case decision might cue the colour decision and accordingly, an additional process would be required to select the case decision as relevant. In contrast, for the univalent stimuli that share no relevant stimulus features with the bivalent stimuli, such as for the univalent stimuli on the parity decision, there is no ambiguity with regard to the task to be performed. Thus, no additional task-decision process would be required. Similar to a negative-priming account, a task-decision account can explain the slowing on tasks with univalent stimuli sharing relevant stimulus features with the bivalent stimuli. However, it cannot explain the slowing on tasks with univalent stimuli sharing no relevant stimulus features with the bivalent stimuli.

Thus, to account for the bivalency effect, we have proposed a different explanation (Meier et al., 2009) which is based on the notion that a stimulus acquires a history during an experiment, i.e., it acquires an association with the task in which it occurs (see Waszak, Hommel, \& Allport, 2003). If episodic binding is not only specific to stimuli and tasks, but also extends to the context in which they occur (i.e., among purely univalent stimuli or among univalent stimuli and occasionally occurring bivalent stimuli), univalent stimuli and tasks might be bound to the more demanding context created by bivalent stimuli. This "episodic context binding" interferes with task performance, irrespective of whether univalent stimuli share or do not share relevant features with the bivalent stimuli, thus resulting in slower performance for all subsequent univalent trials (i.e., the bivalency effect). According to this explanation, the bivalency effect is the result of interference caused by "episodic context binding".

However, recently an alternative explanation has emerged. Based on the theoretical approach that stimulus and response are represented similarly and thus might prime each other (see Hommel, Müsseler, Aschersleben, \& Prinz, 2001), some studies have shown priming from response features to stimulus features (e.g., Deubel \& Schneider, 1996; Fagioli, Hommel, \& Schubotz, 2007; Kunde \& Kiesel, 2006; Metzker \& Dreisbach, 2009; Paprotta, Deubel, \& Schneider, 1999; see Hommel, 2004, for an overview). For instance, Fagioli et al. (2007) reported that when participants prepared a grasping response, they better detected deviants according to their size than according to their location. Conversely, when participants prepared a reaching response, they better detected deviants according to their location than according to their size. Therefore, preparation for a grasping response primed the stimulus features of size and 
preparation for a reaching response primed the stimulus features of location, but not vice versa. This suggests that the activation of response features facilitated the perception of stimulus features, thus providing empirical evidence for priming from response features to stimulus features. A similar conclusion has been reached by Metzker and Dreisbach (2009). They investigated the Simon effect, that is, the performance slowing that occurs when the stimulus position, which is irrelevant for the response, does or does not correspond to the response position. In this study, participants were tested under two instruction conditions. In one condition, the many-to-one mapping condition, they were instructed to respond on the basis of stimulus-response mappings, that is, they were asked to respond to each stimulus with a corresponding response key. In contrast, in the one-to-one mapping condition they were instructed to respond according to a categorization rule. For example, when the name of the presented stimulus started with the letter $\mathrm{B}$, the response was a right key; when it did not start with $\mathrm{B}$, the response was a left key. The results showed a reduced Simon effect when participants responded on the basis of stimulusresponse mappings (many-to-one mapping) compared to the categorization rule (one-to-one mapping). Metzker and Dreisbach (2009) concluded that when a response is mapped to a stimulus according to a categorization rule (one-to-one mapping), the response features strongly prime one single stimulus feature, which results in the Simon effect. In contrast, when a response is directly mapped to several stimuli (many-to-one mapping), the response features prime the stimulus features of several stimuli. As a consequence, the stimulus feature of the relevant stimulus is primed to a smaller degree, thereby reducing the Simon effect. Thus, this study revealed a bidirectional link between stimulus and response features, supporting the view of priming from the response features to stimulus features.

Priming from response features to stimulus features is of particular interest for the bivalency effect as typically, the response features have been identical for all univalent and bivalent stimuli. Based on the evidence that priming can extend from response features to stimulus features (e.g., Deubel \& Schneider, 1996; Fagioli et al., 2007; Kunde \& Kiesel, 2006; Metzker \& Dreisbach, 2009; Paprotta et al., 1999; see Hommel, 2004), it is possible that the response features of univalent stimuli may automatically activate bivalent stimulus features. As a consequence, bivalent stimulus features would have to be inhibited. This inhibition may slow down performance and thus causes the bivalency effect. According to this explanation, the bivalency effect would result from negative priming of bivalent stimulus features via common response features and there would be no need for an explanation in terms of episodic context binding.

The purpose of the present study was to test whether the bivalency effect might result from negative priming of bivalent stimulus features via the response features. To do so, we adapted the paradigm used in the previous studies by systematically varying the overlap of the response sets. During three blocks, participants performed a parity decision on numerals, a colour decision on symbols, and a case decision on letters. Half of the participants used an overlapping response set (i.e., the same two keys for all three tasks), the other half a nonoverlapping response set (i.e., two different keys for each of the three tasks). In the first and third blocks (the purely univalent blocks), all stimuli were univalent. In the second block (the mixed block), some letters for the case decisions appeared coloured which turned them into bivalent stimuli. Stimuli of this kind were evenly distributed across the case decisions.

We hypothesized that if the bivalency effect results from negative priming of bivalent stimulus features via response features (e.g., Fagioli et al., 2007; Metzker \& Dreisbach, 2009; see also Hommel, 2004; Hommel et al., 2001), then it would have a differential impact on the three tasks in the non-overlapping response set relative to the overlapping response set. More precisely, when an overlapping response set is used, the response features are identical for univalent and bivalent stimuli. Thus, the response features of all univalent stimuli would activate the bivalent stimulus features. Consequently, bivalent stimulus features should be inhibited for all univalent stimuli. In this case, performance should be slowed down for all three tasks-replicating the previous results. In contrast, when a non-overlapping response set is used, the response features are identical for univalent and bivalent stimuli in the task in which the bivalent stimuli appear (i.e., the case decision). Thus, the response features would activate the bivalent stimulus features, but only for the case decision. Accordingly, bivalent stimulus features should be inhibited for the case decision and performance should be slowed down for this task only. Alternatively, if the bivalency effect reflects the interference due to episodic context binding (Meier et al., 2009) then univalent stimuli and tasks would be bound to the context that arises from the occasional occurrence of bivalent stimuli. This episodic binding would interfere with performance, irrespective of whether the univalent stimuli share or do not share relevant response features with the bivalent stimuli. According to this explanation, performance should be slowed down for all three tasks independent of the response set. 


\section{Experiment 1}

Method

\section{Participants}

The participants were 40 volunteers (16 men, mean age $=23.2, \mathrm{SD}=2.8$ ) from the University of Bern. Half of them were randomly assigned to the overlapping response set condition and the other half to the non-overlapping response set condition. The study was approved by the local ethical committee of the University of Bern.

\section{Materials}

For the parity decision, the stimuli were the numerals one through eight, each displayed in black. For the colour decision, the stimuli were the symbols $\S, \%$, *, \#, displayed either in blue or red. For the case decision, the stimuli were the consonants $\mathrm{d}, \mathrm{f}, \mathrm{r}, \mathrm{t}$, displayed in black, in either upperor lowercase. We created a set of eight incongruent bivalent stimuli by presenting the same four consonants $(\mathrm{d}, \mathrm{f}, \mathrm{r}$, t) either in blue or red and either in upper- or lowercase. All stimuli were displayed at the centre of the computer screen in a 60-point Times New Roman font.

\section{Procedure}

Participants were tested individually. They were informed that the experiment involved three different tasks: parity decisions about numerals, colour decisions about symbols, and case decisions about letters. Participants with an overlapping response set were instructed to press the keys $b$ or $n$ with their left and right index fingers, respectively, for each of the three tasks. Participants with a non- overlapping response set were instructed to press the keys $b$ or $n$ with their left and right index fingers, respectively, for the parity decision; the keys $v$ or $m$ with their left and right middle fingers, respectively, for the colour decision; the keys $c$ or , with their left and right ring fingers, respectively, for the case decision. The mapping information, printed on paper, was displayed below the computer screen throughout the experiment. Participants were further informed that, for some of the case decisions, the letters would be presented in colour. They were specifically instructed to ignore colour information and to focus on making case decisions.

After these instructions, a block of 30 trial sequences was presented for practice. Each trial sequence required making a parity decision, a colour decision, and a case decision, always in the same order, as illustrated in Fig. 1. The stimulus for each trial was randomly determined and displayed until the participant responded. Then, the screen blanked for $500 \mathrm{~ms}$, and then the next stimulus appeared. After each trial sequence, an additional blank interval of $500 \mathrm{~ms}$ was included. After the practice block and a brief break, each participant completed three experimental blocks without any break between blocks. The first block included 32 trial sequences, with the first two trial sequences serving as "warm-up" sequences which were discarded from the analyses. The second and third blocks had only 30 trial sequences each.

For the first and third blocks (the purely univalent blocks), only univalent stimuli were presented. For the second block (the mixed block), stimuli were univalent, except on $20 \%$ of the case decisions in which bivalent stimuli (i.e., coloured letters) appeared. Bivalent stimuli were determined randomly and without replacement. Trial sequences with bivalent stimuli were evenly interspersed among the 30 trial sequences of the block; occurring in every fifth trial sequence,
Fig. 1 Experiment 1. Example of two consecutive univalent trial sequences. A univalent trial sequence required making a parity decision about numerals, a colour decision about symbols (the symbol \% was written in red and the symbol \# was written in blue), and a case decision about letters. On a bivalent trial sequence (not pictured here), the letters were presented in colour (either in blue or red)

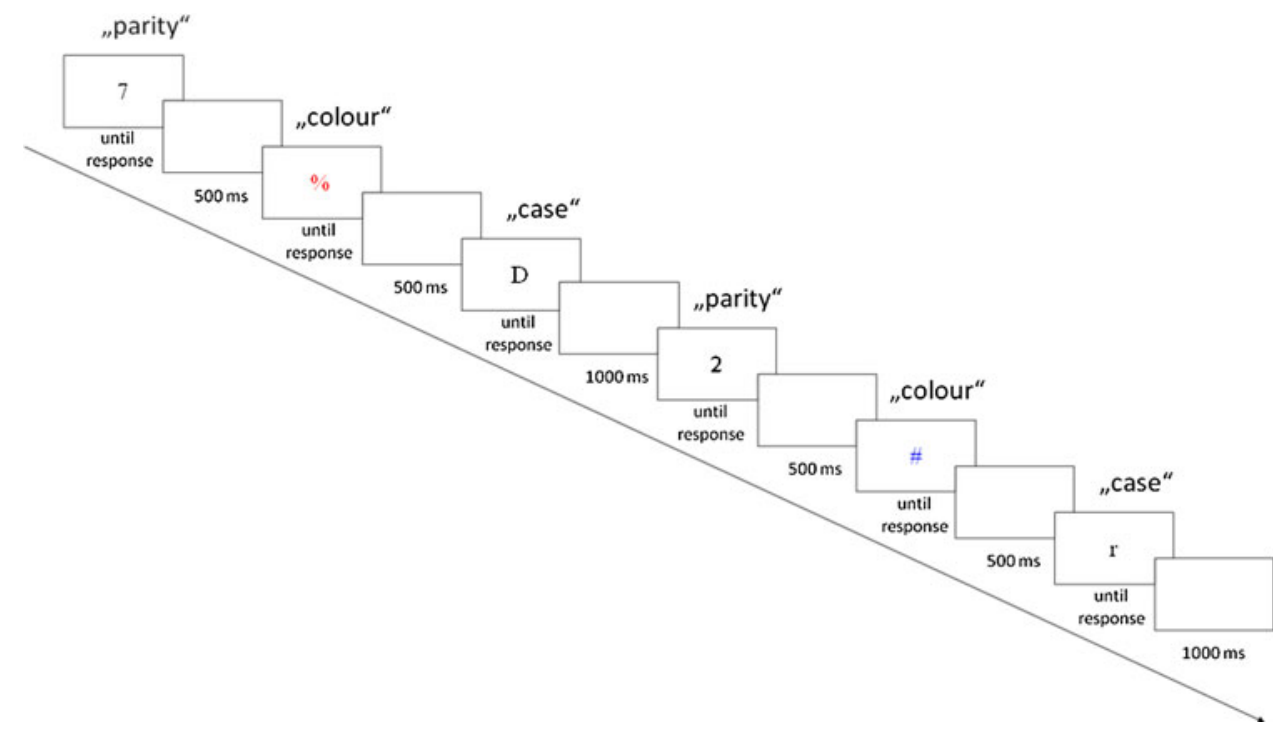


specifically in the 3rd, 8th, 13th, 18th, 23th, and 28th sequences. The entire experiment lasted about $15 \mathrm{~min}$.

\section{Data analysis}

For each participant, the error rates and the median decision times (DTs) for correct responses were computed for each task and each block. For the mixed block, error rates and median DTs for univalent and bivalent case decisions were computed separately. To account for general training effects, we averaged the data from the purely univalent blocks one and three for each task. An alpha level of 0.05 was used for all statistical tests. Greenhouse-Geisser corrections are reported where appropriate and effect sizes are expressed as partial $\eta^{2}$ values.

Results

\section{Performance on bivalent stimuli}

As expected, participants made bivalent case decisions more slowly than univalent case decisions in both response set conditions (overlapping response set: $M_{\text {bivalent }}=1,151 \mathrm{~ms}$, $\mathrm{SE}=113 ; M_{\text {univalent }}=751 \mathrm{~ms}, \mathrm{SE}=50$; and non-overlapping response set: $M_{\text {bivalent }}=1,101 \mathrm{~ms}, \mathrm{SE}=141$; $\left.M_{\text {univalent }}=830 \mathrm{~ms}, \mathrm{SE}=98\right)$. This observation was borne out by a two-factorial analysis of variance (ANOVA) on the case DTs from the mixed block. This ANOVA with stimulus valence (univalent case, bivalent case) as a within-subject factor and response set (overlapping, non-overlapping) as a between-subjects factor showed a significant main effect of stimulus valence, $F(1,38)=34.26, p<0.001, \eta^{2}=0.47$, but no other significant main or interaction effects, $F$ s $<1.26, p \mathrm{~s}>.05, \eta^{2}<0.03$.

The same ANOVA on the accuracy of the case decisions from the mixed block revealed a significant main effect of stimulus valence, $F(1,38)=18.11, p<0.001, \eta^{2}=0.32$, and a significant interaction between stimulus valence and response set, $F(1,38)=6.40, p<0.05, \eta^{2}=0.14$. The main effect of response set was not significant, $F$ (1, 38) $=0.65, p=0.42, \eta^{2}=0.02$. This indicates that participants made more errors on bivalent case decisions than on univalent case decisions, but this difference was more pronounced for the overlapping response set condition than for the non-overlapping response set condition (overlapping response set: $M_{\text {bivalent }}=0.87, \mathrm{SE}=0.03 ; M_{\text {univalent }}=0.99$, SE $<0.01$; and non-overlapping response set: $M_{\text {bivalent }}=$ $\left.0.93, \mathrm{SE}=0.02 ; M_{\text {univalent }}=0.96, \mathrm{SE}=0.01\right)$.

\section{Performance on univalent stimuli}

Figure 2 depicts the means of the median DTs on univalent stimuli with the associated standard errors. Our main

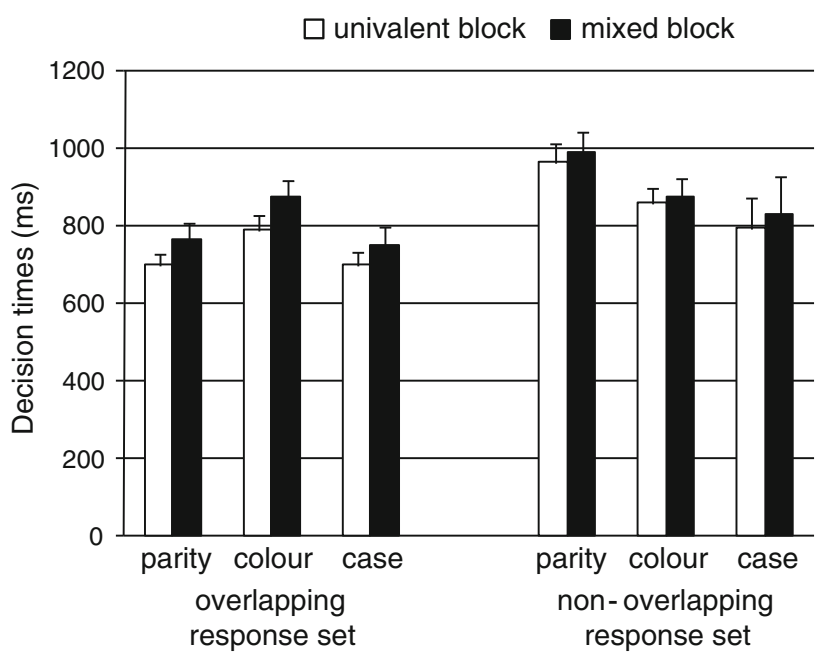

Fig. 2 Experiment 1. Means decision times on trials with univalent stimuli from the purely univalent block (white bars) and those from the mixed block (black bars) in both response set conditions. Error bars represent standard errors. Means are based on median decision times of correct responses out of 30 trials for all conditions, except for the case decisions of the mixed block with only 24 trials

objective was to examine the presence of the bivalency effect in the different tasks across both response set conditions. For this purpose, we carried out a mixed threefactorial ANOVA on the DTs of univalent stimuli, with block (purely univalent, mixed) and task (parity, colour, case) as within-subject factors and response set (overlapping, non-overlapping) as a between-subjects factor. This analysis revealed a significant main effect of block, $F(1$, $38)=11.15, \quad p<0.01, \quad \eta^{2}=0.23$, caused by slower responses on univalent stimuli in the mixed block $(M=847 \mathrm{~ms}, \mathrm{SE}=38)$ than in the purely univalent block $(M=802 \mathrm{~ms}, \mathrm{SE}=32)$. This confirms the presence of the bivalency effect. The analysis also showed a significant main effect of task, $F(2,76)=4.73, p<0.05, \eta^{2}=0.11$, and a significant interaction between task and response set, $F(2,76)=6.14, p<0.01, \eta^{2}=0.14$. This reflects a larger difference between overlapping and non-overlapping response set conditions for parity decisions $(243 \mathrm{~ms})$ than for case decisions $(88 \mathrm{~ms}$ ) and colour decisions ( $35 \mathrm{~ms})$.

Consistent with the episodic context account, the threeway interaction between block, task and response set was not significant, $F(2,76)=0.96, p=0.38, \eta^{2}=0.02$ (observed power for the null effect of the interaction was $0.21)$. Moreover, no other main or interaction effects were significant, $F \mathrm{~s}<3.29, p \mathrm{~s}>0.05, \eta^{2}<0.08$. The bivalency effect was present for all three tasks in both response set conditions. In the overlapping response set condition, it was 62,83 , and $51 \mathrm{~ms}$ for the parity, colour, and case decisions, respectively. In the non-overlapping response set condition, it was 25,17 , and $34 \mathrm{~ms}$, for the parity, colour, and case decisions, respectively. 
Finally, we also carried out a mixed three-factorial ANOVA on the accuracy of univalent stimuli. This ANOVA with block and task as within-subject factors and response set as a between-subjects factor showed a significant main effect of task, $F(1.52,57.75)=8.83$, $p<0.01, \eta^{2}=0.19$. Thus, participants made more errors on parity and colour decisions $(M=0.96, \mathrm{SE}=0.01$; and $M=0.96, \mathrm{SE}=0.01$, respectively) than on case decisions $(M=0.98, \mathrm{SE}=0.00)$. No other main effect or interaction were significant, $F \mathrm{~s}<3.92, p s>0.05, \eta^{2}<0.09$. These results indicate that no speed-accuracy trade-off compromised the critical DTs effects.

\section{Discussion}

The primary goal of Experiment 1 was to test whether the bivalency effect might result from negative priming of bivalent stimulus features via the response features. Therefore, we contrasted task-switching performance on an overlapping and a non-overlapping response set. The results showed a performance slowing on all tasks with univalent stimuli after encountering bivalent stimuli, demonstrating the presence of the bivalency effect in both conditions. This suggests that even if bivalent stimulus features would interfere with the processing of univalent trials that share relevant response features with the bivalent stimuli, this negative priming is not sufficient to explain the bivalency effect. Therefore, the results of Experiment 1 are in line with the view that the bivalency effect stems from the interference caused by episodic context binding rather than being due to negative response set priming.

However, one might argue that the particular set-up of Experiment 1 with the parity decision on the first position may have given rise to a restart effect that might have disguised a potential differential reduction of the bivalency effect. The restart effect refers to slowed performance on the first trial in a trial sequence (Allport \& Wylie, 2000). Therefore, we ran a second experiment to replicate and extend the results from Experiment 1, in which we changed the order of the tasks such that colour became the first and parity the second task. With this change, we were able to make sure that in Experiment 2 any slowing on the parity decision was not caused by a restart effect.

\section{Experiment 2}

Method

\section{Participants}

The participants were 40 different volunteers (24 men, mean age $=24.1, \mathrm{SD}=3.4$ ) from the University of Bern.
As in Experiment 1, half of them were randomly assigned to the overlapping response set condition and the other half to the non-overlapping response set condition.

\section{Materials}

The materials were identical to Experiment 1.

\section{Procedure}

The procedure was also identical to Experiment 1, except that the order of the colour and parity decisions was changed. In Experiment 2, each trial sequence required a colour decision, a parity decision, and a case decision, always in the same order. Participants with an overlapping response set were instructed to press the keys $b$ or $n$ with their left and right index fingers, respectively, for each of the three tasks. Participants with a non-overlapping response set were instructed to press the keys $b$ or $n$ with their left and right index fingers, respectively, for the colour decision; the keys $v$ or $m$ with their left and right middle fingers, respectively, for the parity decision; the keys $c$ or , with their left and right ring fingers, respectively, for the case decision.

\section{Data analysis}

The data analysis was identical to Experiment 1.

Results

\section{Performance on bivalent stimuli}

Participants made bivalent case decisions more slowly than univalent case decisions, but this slowing was more pronounced in the overlapping response set condition $\left(M_{\text {bivalent }}=\right.$ $1,292 \mathrm{~ms}, \mathrm{SE}=144 ; M_{\text {univalent }}=710 \mathrm{~ms}, \mathrm{SE}=33$ ) than in the non-overlapping response set condition $\left(M_{\text {bivalent }}=\right.$ $1,093 \mathrm{~ms}, S E=99 ; M_{\text {univalent }}=831 \mathrm{~ms}, \mathrm{SE}=77$ ). This observation was borne out by a two-factorial ANOVA on the case DTs from the mixed block. This ANOVA with stimulus valence (univalent case, bivalent case) as a within-subject factor and response set (overlapping, non-overlapping) as a between-subjects factor showed a significant main effect of stimulus valence, $F(1,38)=31.39, p<0.001, \eta^{2}=0.45$, and a significant interaction between stimulus valence and response set, $F(1,38)=4.53, p<0.05, \eta^{2}=0.11$. The main effect of response set was not significant, $F(1,38)=0.12$, $p=0.73, \eta^{2}=0.003$.

The same ANOVA on the accuracy of the case decisions from the mixed block revealed a significant main effect of stimulus valence, $F(1,38)=12.93, p<0.01, \eta^{2}=0.25$. No other main or interaction effects were significant, $F_{\mathrm{s}}<0.08, p \mathrm{~s}>0.05, \eta^{2}<0.01$. Thus, participants made 
more errors on bivalent case decisions than on univalent case decisions in both response set conditions (overlapping response set: $M_{\text {bivalent }}=0.92, \mathrm{SE}=0.02 ; M_{\text {univalent }}=0.99$, $\mathrm{SE}<0.01$; and non-overlapping response set: $M_{\text {bivalent }}=$ $0.93, \mathrm{SE}=0.03 ; M_{\text {univalent }}=0.98, \mathrm{SE}=0.01$ ).

\section{Performance on univalent stimuli}

Figure 3 depicts the means of the median DTs on univalent stimuli with the associated standard errors. As in Experiment 1 , our main objective was to examine the presence of the bivalency effect on the different tasks across both response set conditions. We carried out a mixed threefactorial ANOVA with block (purely univalent, mixed) and task (parity, colour, case) as within-subject factors and response set (overlapping, non-overlapping) as a betweensubjects factor. This analysis revealed a significant main effect of block, $F(1,38)=27.96, p<0.001, \eta^{2}=0.42$, caused by slower responses on univalent stimuli in the mixed block $(M=846 \mathrm{~ms}, \mathrm{SE}=32)$ than in the purely univalent block $(M=774 \mathrm{~ms}, \mathrm{SE}=28)$. This confirms the presence of the bivalency effect. The analysis also showed a significant main effect of task, $F$ (1.70, $64.61)=15.00, p<0.001, \eta^{2}=0.28$, and a significant interaction between task and response set, $F$ (1.70, $64.61)=3.45, p<0.05, \eta^{2}=0.08$. This reflects a larger difference between overlapping and non-overlapping response set conditions for parity decisions (105 ms) and case decisions (94 ms) than for colour decisions ( $-4 \mathrm{~ms}$ ).

As in Experiment 1, and consistent with an episodic context binding account, the three-way interaction between

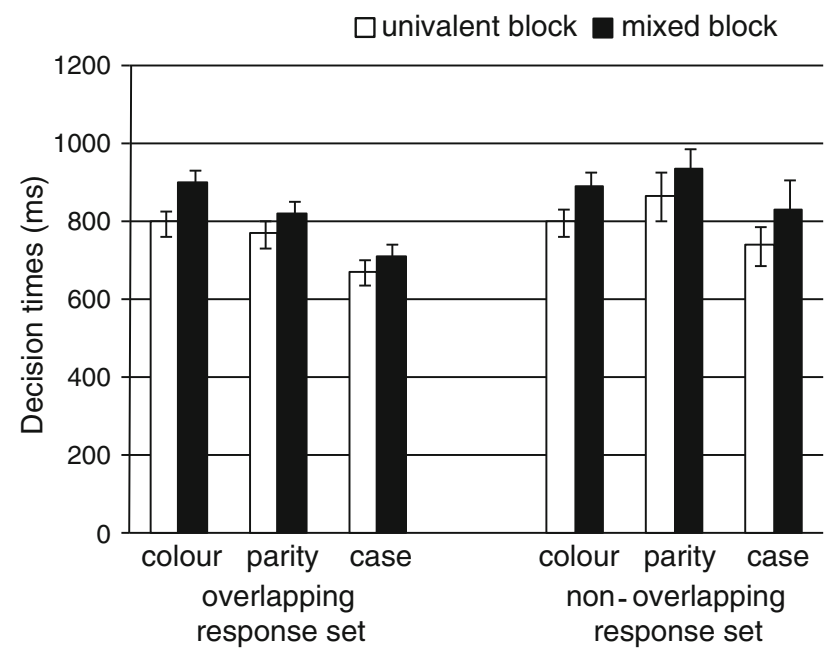

Fig. 3 Experiment 2. Means decision times on trials with univalent stimuli from the purely univalent block (white bars) and those from the mixed block (black bars) in both response set conditions. Error bars represent standard errors. Means are based on median decision times of correct responses out of 30 trials for all conditions except for the case decisions of the mixed block with only 24 trials block, task and response set was not significant, $F$ (1.48, $56.11)=1.41, p=0.25, \eta^{2}=0.04$ (observed power for the null effect of the interaction was 0.25 ). No other main or interaction effects were significant, $F$ s $<1.89$, $p s>0.05, \eta^{2}<0.05$. In the overlapping response set condition, the bivalency effect was 100,48 , and $38 \mathrm{~ms}$ for the colour, parity, and case decisions, respectively. In the non-overlapping response set condition, the bivalency effect was 88, 66, and $93 \mathrm{~ms}$, for the colour, parity, and case decisions, respectively.

Finally, we also carried out a mixed three-factorial ANOVA on the accuracy of univalent stimuli. This ANOVA with block and task as within-subject factors and response set as a between-subjects factor showed a significant main effect of task, $F(1.45,55.19)=15.80$, $p<0.001, \eta^{2}=0.29$. Thus, participants made more errors on colour and parity decisions $(M=0.94, \mathrm{SE}=0.01$; and $M=0.96, \mathrm{SE}=0.01$, respectively) than on case decisions $(M=0.99, \mathrm{SE}=0.00)$. No other main or interaction effects were significant, $F \mathrm{~s}<1.34, p s>0.05, \eta^{2}<0.03$. These results indicate that no speed-accuracy trade-off compromised the critical DTs effects.

\section{Discussion}

The results of Experiment 2 replicated those of Experiment 1. They showed a reliable bivalency effect on all three tasks for both response set conditions. Thus, even when a potential interaction between the bivalency effect and the restart effect was controlled, the findings of the present study revealed a bivalency effect for both response set conditions. This finding again suggests that the bivalency effect is rather due to episodic context binding than to response set priming.

\section{General Discussion}

The purpose of the present study was to test whether the bivalency effect results from negative priming of bivalent stimulus features via common response features, as suggested by recent findings of priming from response features to stimulus features (e.g., Fagioli et al., 2007; Metzker \& Dreisbach, 2009) or whether it stems from episodic context binding (Meier et al., 2009). According to a negative response features priming account, after bivalent stimuli are encountered, bivalent stimulus features would interfere with the processing of univalent trials that share relevant response features with the bivalent stimuli. Thus, bivalent stimulus features would be negatively primed over these trials. This would result in a bivalency effect for the univalent trials that share relevant response features with the bivalent stimuli. In contrast, according to an episodic 
context binding account, univalent stimuli and tasks would be bound to the context in which bivalent stimuli have been encountered occasionally. This "episodic context binding" would interfere with processing all subsequent trials, thus causing a bivalency effect irrespective of the response set overlap between univalent and bivalent stimuli.

In two experiments, we found a performance slowing on univalent trials after bivalent stimuli were occasionally presented. More critically, this slowing - the bivalency effectwas found independent of whether or not univalent and bivalent stimuli shared the same response sets. This finding rules out the possibility that the bivalency effect simply arises from negative priming of bivalent stimulus features via common response features (e.g., Fagioli et al., 2007; Metzker \& Dreisbach, 2009). In fact, such an explanation cannot explain why the bivalency effect was found for tasks with response features different from those of the bivalent stimuli (i.e., for parity and colour decisions in the non-overlapping response set) ${ }^{1}$.

\footnotetext{
${ }^{1}$ One could argue that there may still be a potential overlap in the non-overlapping response set depending on how responses are coded (see Druey \& Hübner, 2008; Hübner \& Druey, 2008). For example, participants in the non-overlapping response set condition might code responses according to the anatomical features of the finger types or according to the spatial features of the response keys. Accordingly, they would have coded the responses according to the three finger types (i.e., index, middle, ring) or to the spatial features of the response keys (i.e., left, middle, right). If participants would code responses according to the three finger types, the index, middle, and ring fingers for both hands would be mapped to the parity, colour, and case decisions, respectively, in Experiment 1, and to the colour, parity, and case decisions, respectively, in Experiment 2. As bivalent stimuli always appeared in the case decisions, bivalent stimulus features would be linked to the response features of the ring fingers. However, they would never be linked to the response features of the index or middle fingers. As a consequence, even when responses are coded according to anatomical features of the finger types, the response set would not overlap between univalent and bivalent stimuli for the parity and colour decisions.

Alternatively, if participants would code responses according to the spatial features of the response keys, the response features left, middle, and right would be mapped in Experiment 1 to the case, colour, and parity decisions, respectively, for the left hand and to the parity, colour, and case decisions, respectively, for the right hand. Similarly, in Experiment 2, the response features left, middle, and right would be mapped to the case, parity, and colour decisions, respectively, for the left hand and to the colour, parity, and case decisions, respectively, for the right hand. In this case, bivalent stimulus features would be linked to the response features left and right. Therefore, the response set would overlap between univalent and bivalent stimuli for the parity and case decisions in Experiment 1 and for the colour and case decisions in Experiment 2. In contrast, it would never overlap for the colour decision in Experiment 1 and for the parity decision in Experiment 2 as these tasks were mapped to the response feature middle. Accordingly, even if participants would have coded responses according to the spatial features of the response keys, the response set would still not overlap for at least one of the three tasks. Therefore, it does not matter how the responses are coded because for both types of response codes (finger vs. spatial), negative priming of bivalent stimulus features via common response features would not be sufficient to account for the present results.
}

In addition, the present study also replicated the previous findings (Meier et al., 2009; Woodward et al., 2003; 2008) by showing a similar bivalency effect on tasks with univalent stimuli sharing relevant stimulus features with the bivalent stimuli and on those with univalent stimuli sharing no relevant stimulus features with the bivalent stimuli. Taken together, the findings of the present study showed that negative priming accounts operating across either stimulus or response features are not sufficient to explain the bivalency effect (cf., Allport et al., 1994; Allport \& Wylie, 1999; 2000; Wylie \& Allport, 2000). Furthermore, they suggest that the task-decision process accounts must be extended in order to explain the bivalency effect (cf., Fagot, 1994; Meiran et al., 2008; Monsell et al., 2000; Rogers \& Monsell, 1995; Rubinstein, et al., 2001; Sohn \& Anderson, 2001). For instance, using univalent stimuli Braverman and Meiran (2010) provided evidence for a task-decision process that involves stimulusand task-relevant features. Thus, if the task-decision process would not only involve stimulus- or task-relevant features but also context-relevant features i.e., episodic context binding, then it would be possible to account for the bivalency effect.

Thus, the results of the present study revealed that the adjustment of cognitive control involved in the bivalency effect could not be the result of stimulus priming or of response priming. This finding is of particular interest for the current debate in cognitive control research as to whether the cognitive control effects are related to cognitive control at all, or whether they are the result of priming processes operating across stimulus or response representations (see Egner, 2007, for an overview). This debate has focused on a paradigm that mixes congruent stimuli (i.e., stimuli affording the same response) with incongruent stimuli (i.e., stimuli affording different responses). In these paradigms, congruency effects (i.e., the performance slowing on incongruent stimuli relative to congruent stimuli) are usually smaller after an incongruent stimulus than after a congruent stimulus. Typically, this sequential modulation of congruency effects has been interpreted as a result from an adjustment of cognitive control (e.g., Botvinick et al., 2001). According to this account, as an incongruent stimulus leads to the activation of two responses, it involves a conflict. In order to overcome this conflict, an adjustment of cognitive control is required. Interestingly, this adjustment of cognitive control persists across the subsequent trial. This facilitates processing when the stimulus in the subsequent trial is incongruent. In contrast, when the stimulus in the first trial is congruent, less cognitive control is necessary and this impairs processing when the stimulus in the subsequent trial is incongruent. Taken together, this explains the sequential modulation of congruency effects. 
Recently, however, this sequential modulation is suggested to be mediated by priming effects (e.g., Hommel, 2004; Mayr, Awh, \& Laurey, 2003). More precisely, in congruent trials following congruent trials and in incongruent trials following incongruent trials, all stimulus and response features are repeated or all features change. Thus, in complete repetitions, repetition priming might occur and in complete changes, no negative priming at all might occur. In both cases, this would result in a performance benefit. In contrast, in congruent trials following incongruent trials and in incongruent trials following congruent trials, one feature (stimulus or response) changes and the others remain the same. Thus, in these partial repetitions, no repetition priming should occur, whereas negative priming might occur. In this case, performance should slow down. However, although this account is consistent with the sequential modulation of congruency effects, it cannot entirely explain the findings of the present study that showed a bivalency effect, irrespective of stimulus or response priming. Thus, some cognitive control effects, such as the bivalency effect, might be related to cognitive control, and others, such as the sequential modulation of congruency effects, might be the result of priming processes.

The results of the present study are rather compatible with the explanation that the adjustment of cognitive control involved in the bivalency effect reflects the interference caused by episodic context binding. Based on the evidence that experiencing a stimulus in a specific task affects performance (see Waszak et al., 2003), we have proposed that experiencing stimuli or tasks in a specific context, such as the more difficult demand of occasionally encountering bivalent stimuli, can also affect performance (Meier et al., 2009). In this way, performance results from episodic binding that involves not only stimuli or tasks but also the context in which they occur. This notion is consistent with the recent findings from Waszak and Pholulamdeth (2009) who observed that an emotionally arousing picture modulates the episodic binding between a stimulus and a task. However, the results of the present study suggest that a context does not even need to be emotionally arousing to have an impact on performance. It is sufficient when it triggers specific demands, such as the increased demands caused by occasionally encountering bivalent stimuli.

Summing up, the findings of the present study showed that the bivalency effect was similar when univalent and bivalent stimuli shared the same response features and when they did not. Furthermore, it was also similar for tasks with univalent stimuli sharing relevant stimulus features with the bivalent stimuli and for those with univalent stimuli sharing no relevant stimulus features with the bivalent stimuli. The adjustment of cognitive control involved in the bivalency effect, therefore, does not arise from negative priming of bivalent stimulus features. Rather, the results support the view that the bivalency effect reflects the interference caused by episodic context binding.

Acknowledgments This work was supported by a grant from the Janggen-Pöhn Foundation to A. Rey-Mermet and by a grant from the Swiss National Science Foundation (Grant 130104) to B. Meier. We thank Martina Godosev, Cornelia Häfliger, Sara Mössinger, Isabelle Lüdhi, and Jona Hermann for testing the participants, as well as Brigitte Weiermann, Nicolas Rothen and Josephine Cock for helpful comments on an earlier version.

\section{References}

Allport, A., Styles, E. A., \& Hsieh, S. (1994). Shifting intentional set: Exploring the dynamic control of tasks. In C. Umilta \& M. Moscovitch (Eds.), Attention and performance XV: conscious and non-conscious information processing (pp. 421-452). Cambridge: MIT.

Allport, A., \& Wylie, G. (1999). Task-switching: positive and negative priming of task-set. In G. W. Humphreys, J. Duncan, \& A. M. Treisman (Eds.), Attention, space and action: Studies in cognitive neuroscience (pp. 273-296). Oxford: Oxford University Press.

Allport, A., \& Wylie, G. (2000). Task switching, stimulus-response bindings, and negative priming. In S. Monsell \& J. S. Driver (Eds.), Control of cognitive processes: Attention and performance XVIII (pp. 35-70). Cambridge: MIT.

Botvinick, M. M., Braver, T. S., Barch, D. M., Carter, C. S., \& Cohen, J. D. (2001). Conflict monitoring and cognitive control. Psychol Rev, 108, 624-652.

Braverman, A., \& Meiran, N. (2010). Task conflict in task switching. Psychol Res, 74, 568-578.

Deubel, H., \& Schneider, W. X. (1996). Saccade target selection and object recognition: Evidence for a common attentional mechanism. Vis Res, 36, 1827-1837.

Druey, M. D., \& Hübner, R. (2008). Effects of stimulus features and instruction on response coding, selection, and inhibition: evidence from repetition effects under task switching. $Q J$ Exp Psychol, 61, 1573-1600.

Egner, T. (2007). Congruency sequence effects and cognitive control. Cogn Affect Behav Neurosci, 7, 380-390.

Fagioli, S., Hommel, B., \& Schubotz, R. I. (2007). Intentional control of attention: Action planning primes action-related stimulus dimensions. Psychol Res, 71, 22-29.

Fagot, C. (1994). Chronometric investigations of task switching. Unpublished doctoral dissertation, San Diego: University of California.

Hommel, B. (2004). Event files: Feature binding in and across perception and action. Trends Cogn Sci, 8, 494-500.

Hommel, B., Müsseler, J., Aschersleben, G., \& Prinz, W. (2001). The theory of event coding (TEC): A framework for perception and action planning. Behav Brain Sci, 24, 849-937.

Hübner, R., \& Druey, M. D. (2008). Multiple response codes play specific roles in response selection and inhibition under task switching. Psychol Res, 72, 415-424.

Jersild, A. T. (1927). Mental set and shift. Arch Psychol, 89, 5-82.

Kunde, W., \& Kiesel, A. (2006). See what you've done! Active touch affects the number of perceived visual objects. Psychon Bull Rev, 13, 304-309. 
Mayr, U., Awh, E., \& Laurey, P. (2003). Conflict adaptation effects in the absence of executive control. Nat Neurosci, 6, 450-452.

Meier, B., Woodward, T. S., Rey-Mermet, A., \& Graf, P. (2009). The bivalency effect in task switching: General and enduring. Can J Exp Psychol, 63, 201-210.

Meiran, N. (2008). The dual implication of dual affordance: Stimulustask binding and attentional focus of changing during task preparation. Exp Psychol, 55, 251-259.

Meiran, N., Kessler, Y., \& Adi-Japha, E. (2008). Control by action representation and input selection (CARIS): A theoretical framework for task switching. Psychol Res, 72, 473-500.

Metzker, M., \& Dreisbach, G. (2009). Bidirectional priming processes in the Simon task. J Exp Psychol Hum Percept Perform, 35, $1770-1783$.

Monsell, S., Yeung, N., \& Azuma, R. (2000). Reconfiguration of taskset: Is it easier to switch to the weaker task? Psychol Res, 63, 250-264.

Paprotta, I., Deubel, H., \& Schneider, W. X. (1999). Object recognition and goal-directed eye or hand movements are coupled by visual attention. In W. Becker, H. Deubel, \& T. Mergner (Eds.), Current oculomotor research: physiological and psychological aspects (pp. 241-248). New York: Plenum.

Rogers, R. D., \& Monsell, S. (1995). Costs of a predictable switch between simple cognitive tasks. J Exp Psychol Gen, 124, 207-231.
Rubinstein, J. S., Meyer, D. E., \& Evans, J. E. (2001). Executive control of cognitive processes in task switching. J Exp Psychol Hum Percept Perform, 27, 763-797.

Sohn, M.-H., \& Anderson, J. R. (2001). Task preparation and task repetition: Two-component model of task switching. $J$ Exp Psychol Gen, 130, 764-778.

Steinhauser, M., \& Hübner, R. (2009). Distinguishing response conflict and task conflict in the Stroop task: Evidence from ex-Gaussian distribution analysis. J Exp Psychol Hum Percept Perform, 35, 1398-1412.

Waszak, F., Hommel, B., \& Allport, A. (2003). Task-switching and long-term priming: Role of episodic stimulus-task bindings in task-shift costs. Cogn Psychol, 46, 361-413.

Waszak, F., \& Pholulamdeth, V. (2009). Episodic S-R bindings and emotion: About the influence of positive and negative effects on stimulus-response associations. Exp Brain Res, 194, 489-494.

Woodward, T. S., Meier, B., Tipper, C., \& Graf, P. (2003). Bivalency is costly: Bivalent stimuli elicit cautious responding. Exp Psychol, 50, 233-238.

Woodward, T. S., Metzak, P. D., Meier, B., \& Holroyd, C. B. (2008). Anterior cingulate cortex signals the requirement to break inertia when switching tasks: A study of the bivalency effect. Neuroimage, 40, 1311-1318.

Wylie, G., \& Allport, A. (2000). Task switching and the measurement of "switch costs". Psychol Res, 63, 212-233. 